\title{
TOPOMORPHISM - A NEW APPROACH TO IDENTIFY TOPOLOGIES
}

\author{
Ramachandran Vembu \\ Department of Mathematics, SBK College, Aruppukottai - 626 101, India \\ *Corresponding author, e-mail: msrvembu@yahoo.co.in
}

\begin{abstract}
A new concept called topomorphism is defined and discussed in this paper. A topomorphism is defined as a bijection between topologies which preserve finite intersection and arbitrary union. It is proved that topomorphisms preserve connectedness and compactness. Topomorphisms in the context of separation axioms and the role of bases in topomorphism were studied deeply.
\end{abstract}

Key words: topology; homeomorphism; topomormhism; identification of topologies; connectedness; compactness; separation axioms

\section{INTRODUCTION}

Let us start with the topological spaces $(X, \mathcal{T})$ and $\left(Y, \mathcal{T}^{\prime}\right)$ where $X=\{1,2,3, \ldots, 10\}, \quad \mathrm{Y}=$ $\{1,2,3, \ldots, 20\} \quad, \quad \mathcal{T}=\{X, \varnothing,\{1,2,3\}\}$ and $\mathcal{T}^{\prime}=$ $\{Y, \emptyset,\{1,2,3,4,5,6\}\}$. These two topological spaces are not homeomorphic as there is no bijection between $X$ and $Y$. But one can feel that these two spaces are the same in some sense. This feeling motivates us to define the concept of topomorphism. A topomorphism from a topological space $(X, \mathcal{T})$ to a topological space $\left(Y, \mathcal{T}^{\prime}\right)$ is defined as a bijection from $\mathcal{T}$ to $\mathcal{T}^{\prime}$ preserving finite intersection and arbitrary union. In this paper we define and discuss the concept of topomorphism.

In Section 2 we give all the definitions and notations which we use in this paper, in Section 3 we define topomorphism and investigate certain properties, in Section 4 we study the relationship between topomorphism and homeomorphism, in Section 5 we discuss the concepts of compact subsets and connected subsets of a topological space in the context of topomorphism and in Section 6 we discuss topomorphism in the context of bases for topologies; finally we end the paper with a few concluding remarks motivating further research in this area.

\section{DEFINITIONS AND RESULTS}

First we fix the notations. Let $\mathbb{R}$ denote the set of real numbers, $\varnothing$ denote the empty set. We use the notation $E^{c}$ to denote the complement of $E$ in the corresponding space unless there is no ambiguity. For sets $A$ and $B$, by $A-B$ we denote the set $A \cap$ $B^{c}$. If $F: \mathcal{T} \rightarrow \mathcal{T}^{\prime}$ is a bijection, then for $B \in \mathcal{T}^{\prime}$, by $F^{-1}(B)$ we denote the unique element $A \in \mathcal{T}$ such that $F(A)=B$. For any function $f: X \rightarrow Y$, if $A \subseteq$ $X$ and $B \subseteq Y$, by $f^{\rightarrow}(A)$ and $f^{\leftarrow}(B)$ we denote the sets $\{f(a) / a \in A\}$ and $\{a \in A / f(a) \in B\}$ respectively. These sets are usually denoted by $f(A)$ and $f^{-1}(\mathrm{~B})$; if $F$ and $f$ are a topomorphism and a homeomorphism from $(X, \mathcal{T})$ to $\left(Y, \mathcal{T}^{\prime}\right)$, to avoid confusions (for example between $F(A)$, the image of the element $A$ in $\mathcal{T}$ and $f(A)$, the set of all images of points in $A$ ) we use these notations.

A bijective open continuous function from a topological space to a topological space is called a homeomorphism. For terminologies and notations used in this paper which are not mentioned here we refer to [1]. However it is worthwhile to state that we assume one point sets are closed in regular spaces and normal spaces. 


\section{Topomorphism}

We start with the definition of a topomorphism.

Definition 3.1. Let $(X, \mathcal{T})$ and $\left(Y, \mathcal{T}^{\prime}\right)$ be topological spaces. A bijective function $F: \mathcal{T} \rightarrow \mathcal{T}^{\prime}$ is said to be a topomorphism from $(X, \mathcal{T})$ and $\left(Y, \mathcal{T}^{\prime}\right)$ if

i. $\quad F\left(\cup A_{\alpha}\right)=\cup F\left(A_{\alpha}\right)$ for any collection $\left\{A_{\alpha}\right\}$ of elements of $\mathcal{T}$.

ii. $\quad F(A \cap B)=F(A) \cap F(B)$ for any elements $A$ and $B$ of $\mathcal{T}$.

If there is a topomorphism from $(X, \mathcal{T})$ to $\left(Y, \mathcal{T}^{\prime}\right)$, we say, as usual, $\left(Y, \mathcal{T}^{\prime}\right)$ is topomorphic to $(X, \mathcal{T})$.

Example 3.2. On $\mathbb{R}$, consider the topologies $\mathcal{T}_{1}=\{\varnothing, \mathbb{R},(0,1),(0,2)\}, \mathcal{T}_{2}=\{\varnothing, \mathbb{R},\{3\},\{3,4\}\}$ and $\mathcal{T}_{3}=\{\varnothing, \mathbb{R},(-\infty, 0],(0, \infty)\}$. The mapping $F: \mathcal{T}_{1} \rightarrow \mathcal{T}_{2}$, taking the sets $\emptyset, \mathbb{R},(0,1),(0,2)$ to $\emptyset, \mathbb{R},\{3\},\{3,4\}$ respectively, is a topomorphism whereas there is no topomorphism from $\mathcal{T}_{1}$ to $\mathcal{T}_{3}$.

Theorem 3.3. Let $(X, \mathcal{T})$ and $\left(Y, \mathcal{T}^{\prime}\right)$ be topological spaces and let $F: \mathcal{T} \rightarrow \mathcal{T}^{\prime}$ be a topomorphism. Then

i. For $A, B \in \mathcal{T}$, if $A \subseteq B$, then $F(A) \subseteq$ $F(B)$ and if $A$ is strictly contained in $B$, then $F(A)$ is strictly contained in $F(B)$.

ii. $\quad F(X)=Y$ and $F(\varnothing)=\varnothing$.

Proof. Let $A \subseteq B$. Then $A \cup B=B$ and hence $F(A \cup B)=F(B)$ which implies that $F(A) \cup$ $F(B)=F(B)$; hence $F(A) \subseteq F(B)$. This proves the first part of (i); as $F$ is a bijection, the second part of (i) follows.

Let $U=F^{-1}(\mathrm{Y})$. Then $F(U)=Y$. As $U \subseteq X$, $F(U) \subseteq F(X)$ and hence $F(X)=Y$. Simi$\operatorname{larly} F(\varnothing)=\varnothing$.

Theorem 3.4. The composition of topomorphisms is a topomorphism and the inverse of a topomorphism is a topomorphism.

Proof. Let $F_{1}:\left(X_{1}, \mathcal{T}_{1}\right) \rightarrow\left(X_{2}, \mathcal{T}_{2}\right)$ and $F_{2}:\left(X_{2}, \mathcal{T}_{2}\right) \rightarrow\left(X_{3}, \mathcal{T}_{3}\right)$ be topomorphisms. Let the composition $F_{2} \circ F_{1}:\left(X_{1}, \mathcal{T}_{1}\right) \rightarrow\left(X_{3}, \mathcal{T}_{3}\right)$ be denoted by $F$. As $F_{1}$ and $F_{2}$ are bijections, $F$ is also a bijection. Let $\left\{A_{\alpha}\right\}$ be a collection of elements of $\mathcal{T}_{1}$. Now

$$
\begin{gathered}
F\left(\cup A_{\alpha}\right)=F_{2}\left(F_{1}\left(\cup A_{\alpha}\right)\right)=F_{2}\left(\cup F_{1}\left(A_{\alpha}\right)\right) \\
=\cup F_{2}\left(F_{1}\left(A_{\alpha}\right)\right)=\cup F\left(A_{\alpha}\right)
\end{gathered}
$$

and similarly

$$
F(A \cap B)=F(A) \cap F(B) .
$$

Thus $F$ is a topomorphism.

To prove the second part, let $F:(X, \mathcal{T}) \rightarrow$ $\left(Y, \mathcal{T}^{\prime}\right)$ be a topomorphism. As $F$ is a bijection, its inverse $F^{-1}:\left(Y, \mathcal{T}^{\prime}\right) \rightarrow(X, \mathcal{T})$ exists and is a bijection. Let $\left\{B_{\alpha}\right\}$ be a collection of elements of $\mathcal{T}^{\prime}$. For each $\alpha$, let $A_{\alpha}=F^{-1}\left(B_{\alpha}\right)$. Then $B_{\alpha}=F\left(A_{\alpha}\right)$ and $F\left(\cup A_{\alpha}\right)=\cup F\left(A_{\alpha}\right)$. Thus $F\left(\cup A_{\alpha}\right)=\cup B_{\alpha}$ and hence $\cup A_{\alpha}=F^{-1}\left(\cup B_{\alpha}\right)$ which in turn gives

$$
F^{-1}\left(\cup B_{\alpha}\right)=\cup F^{-1}\left(B_{\alpha}\right) \text {. }
$$

Let $B_{1} . B_{2}$ be elements of $\mathcal{T}^{\prime}$. For $i=1,2$, let $A_{i}=F^{-1}\left(B_{i}\right)$. Then as $F$ is a topomorphism, we have $F\left(A_{1} \cap A_{2}\right)=F\left(A_{1}\right) \cap F\left(A_{2}\right)$. So $F\left(A_{1} \cap\right.$ $\left.A_{2}\right)=B_{1} \cap B_{2}$ and hence $A_{1} \cap A_{2}=F^{-1}\left(B_{1} \cap\right.$ $B_{2}$ ) which implies that

$$
F^{-1}\left(B_{1} \cap B_{2}\right)=F^{-1}\left(B_{1}\right) \cap F^{-1}\left(B_{2}\right) \text {. }
$$

Thus $F^{-1}$ is a topomorphism.

It is easy to see that the identity map from $\mathcal{T}$ to $\mathcal{T}$ is a topomorphism and hence by Theorem 3.4, the relation $\sim$ defined on the class of all topological spaces by " $(X, \mathcal{T}) \sim\left(Y, \mathcal{T}^{\prime}\right)$ if there is a topomorphism from $(X, \mathcal{T})$ to $\left(Y, \mathcal{T}^{\prime}\right)$ " is an equivalence relation.

Theorem 3.5. Let $F:(X, \mathcal{T}) \rightarrow\left(Y, \mathcal{T}^{\prime}\right)$ be a topomorphism. Then

i. $(X, \mathcal{T})$ is connected if and only if $\left(Y, \mathcal{T}^{\prime}\right)$ is connected.

ii. $\quad(X, \mathcal{T})$ is compact if and only if $\left(Y, \mathcal{T}^{\prime}\right)$ is compact.

Proof. Let $(X, \mathcal{T})$ be connected. Let us assume that $\left(Y, \mathcal{T}^{\prime}\right)$ is not connected. Then there are nonempty sets $B_{1}, B_{2} \in \mathcal{T}^{\prime}$ such that

$$
B_{1} \cup B_{2}=Y \text { and } B_{1} \cap B_{2}=\varnothing .
$$

Let

$$
A_{1}=F^{-1}\left(B_{1}\right) \text { and } A_{2}=F^{-1}\left(B_{2}\right) \text {. }
$$

As $B_{1}$ and $B_{2}$ are nonempty sets, $A_{1}$ and $A_{2}$ are nonempty sets. Now

$$
\begin{gathered}
F\left(A_{1} \cup A_{2}\right)= \\
=F\left(A_{1}\right) \cup F\left(A_{2}\right)=B_{1} \cup B_{2}=Y \\
=F(X)
\end{gathered}
$$

and

$$
\begin{aligned}
F\left(A_{1} \cap A_{2}\right)= & F\left(A_{1}\right) \cap F\left(A_{2}\right)=B_{1} \cap B_{2}=\varnothing \\
& =F(\varnothing)
\end{aligned}
$$

Hence, as $F$ is a bijection,

$$
A_{1} \cup A_{2}=X \text { and } A_{1} \cap A_{2}=\varnothing
$$

This is a contradiction proving a part of (i).

Let $(X, \mathcal{T})$ be compact. Let $\left\{B_{\alpha}\right\}$ be an open cover for $Y$. Let $A_{\alpha}=F^{-1}\left(B_{\alpha}\right)$. Then 


$$
F\left(\cup A_{\alpha}\right)=\cup F\left(A_{\alpha}\right)=\cup B_{\alpha}=Y=F(X) .
$$

and hence $\cup A_{\alpha}=X$. Thus $\left\{A_{\alpha}\right\}$ is an open cover for $X$. As $X$ is compact, this open cover has a finite subcover, say $\left\{A_{1}, A_{2}, \ldots, A_{n}\right\}$. Then $\left\{B_{1}, B_{2}, \ldots, B_{n}\right\}$ is a finite subcover of $\left\{B_{\alpha}\right\}$ proving a part of (ii). Since $F^{-1}$ is also a topomorphism, converse parts follow.

\section{Topomorphisms and Homeomorphisms}

Let us now see a relation between topomorphism and homeomorphism.

Definition 4.1. Let $f:(X, \mathcal{T}) \rightarrow\left(Y, \mathcal{T}^{\prime}\right)$ be a homeomorphism. Let $F: \mathcal{T} \rightarrow \mathcal{T}^{\prime}$ be defined as

$$
F(A)=f^{\rightarrow}(A) .
$$

Then $F$ is a topomorphism and is called the topomorphism induced by the homeomorphism $f$.

As a homeomorphism takes open sets to open sets, the function $F$ is well defined; since $f$ is a bijection and $f^{\leftarrow}(B)$ is open in $X$ whenever $B$ is open in $Y$, we see that $F$ is a bijection. Now

$$
F\left(\cup A_{\alpha}\right)=f^{\rightarrow}\left(\cup A_{\alpha}\right)=\cup f^{\rightarrow}\left(A_{\alpha}\right)=\cup F\left(A_{\alpha}\right)
$$

and

$$
\begin{gathered}
F(A \cap B)=f^{\rightarrow}(A \cap B)=f^{\rightarrow}(A) \cap f^{\rightarrow}(B) \\
=F(A) \cap F(B) .
\end{gathered}
$$

Thus Definition 4.1 is well-defined.

Though every homeomorphism induces a topomorphism, not all topomorphisms are induced by homeomorphisms, even if the two topological spaces have the same cardinality; the topomorphism $F$ of Example 3.2 is not induced by any homeomorphism as $f^{\leftarrow}(\{3\})$ cannot be an uncountable set under any bijection. Thus the concept of topomorphism is a proper generalization of homeomorphism.

Topomorphism will not preserve the separation properties like Hausdorff and regular as seen in the following example.

\section{Example 4.2.}

Let $X=\{1,2\}, \mathcal{T}=\{\varnothing, X,\{1\},\{2\}\}, Y=\mathbb{R}$ and $\mathcal{T}^{\prime}=\{\varnothing, \mathbb{R},(-\infty, 0],(0, \infty)\}$. Then $(X, \mathcal{T})$ and $\left(Y, \mathcal{T}^{\prime}\right)$ are topomorphic, $(X, \mathcal{T})$ is Hausdorff, regular and normal whereas $\left(Y, \mathcal{T}^{\prime}\right)$ is not even a Hausdorff space.

But if one point sets are closed in one of the two spaces between which a topomorphism exists, then we get interesting properties.

Theorem 4.3 Let $F:(X, \mathcal{T}) \rightarrow\left(Y, \mathcal{T}^{\prime}\right)$ be a topomorphism and let one point sets in $(X, \mathcal{T})$ be closed. Then $(X, \mathcal{T})$ is homeomorphic to a subspace of $\left(Y, \mathcal{T}^{\prime}\right)$.

Proof. Define a function $f: X \rightarrow Y$ as follows:

For any $a \in X$, as $\{a\}$ is closed in $(X, \mathcal{T}), X-$ $\{a\} \in \mathcal{T}$. As $X-\{a\} \subsetneq X, \quad F(X-\{a\}) \varsubsetneqq F(X)$. Choose an element in $y \notin F(X-\{a\})$ and declare it as $f(a)$. (This is possible by the axiom of choice.)

We first prove that $F(X-\{a\})$ is the largest open set not containing $f(a)$. There is no open set strictly between $X-\{a\}$ and $X$. Hence, by Theorem 3.3 , there is no open set strictly between $F(X-\{a\})$ and $F(X)$. As $F(X)=Y, F(X)$ contains $f(a)$. Hence $F(X-\{a\})$ is not contained in any larger open set not containing $f(a)$. Furthermore the union of all open sets not containing $f(a)$ (say $V_{a}$ ) is clearly the largest open set not containing $f(a)$. But then $F(X-\{a\}) \subseteq V_{a}$ and hence $F(X-\{a\})=V_{a}$. Thus $F(X-\{a\})$ is the largest open set not containing $f(a)$.

We now prove that $f$ is one-to-one. Let $a_{1}, a_{2} \in X$ be such that $a_{1} \neq a_{2}$. Let $V_{1}=F(X-$ $\left.\left\{a_{1}\right\}\right)$ and $V_{2}=F\left(X-\left\{a_{2}\right\}\right)$. By definition $f\left(a_{1}\right) \notin V_{1}$. We claim that $f\left(a_{1}\right) \in V_{2}$. If $f\left(a_{1}\right) \notin$ $\mathrm{V}_{2}$, then $V_{1} \cup V_{2} \subsetneq Y$; hence

$$
\begin{gathered}
F(X)=F\left(\left(X-\left\{a_{1}\right\}\right) \cup\left(X-\left\{a_{2}\right\}\right)\right. \\
=F\left(X-\left\{a_{1}\right\}\right) \cup F\left(X-\left\{a_{2}\right\}\right)=V_{1} \cup V_{2} \varsubsetneqq Y .
\end{gathered}
$$

This is a contradiction to the fact that $F(X)=$ $Y$. So $f\left(a_{1}\right) \in V_{2}$. But $f\left(a_{2}\right) \notin \mathrm{V}_{2}$. Thus $f\left(a_{1}\right) \neq$ $f\left(a_{2}\right)$ and hence $f$ is one-to-one.

Now let

$$
Y^{*}=\{f(a) / a \in X\} .
$$

Then $Y^{*}$ is a subset of $Y$ and $f$ is a bijection from $X$ to $Y^{*}$. Let

$$
\mathcal{T}^{*}=\left\{f^{\rightarrow}(U) / U \in \mathcal{T}\right\} .
$$

We now prove that $\mathcal{T}^{*}=\left\{V \cap Y^{*} / V \in \mathcal{T}^{\prime}\right\}$ which will imply that $\left(Y^{*}, \mathcal{T}^{*}\right)$ is a subspace of $(Y, \mathcal{T})$.

Let $W \in \mathcal{T}^{*}$. Then $W=f^{\rightarrow}(U)$ for some $U \in \mathcal{T}$. Since $f$ is a bijection, $U$ is unique. We claim that $W=F(U) \cap Y^{*}$. Let $b \in W$. Then $b=$ $f(a)$ for some $a \in U$. Suppose that $f(a) \notin F(\mathrm{U})$. Since $F(X-\{a\})$ is the largest open subset of $Y$ not containing $f(a)$ and $F(U)$ is an open subset of $Y$ not containing $f(a)$ we have $F(U) \subseteq F(X-$ $\{a\})$ and hence $U \subseteq X-\{a\}$. This is a contradiction to the fact that $a \in U$. Thus $f(a) \in F(U)$ and hence $b \in F(U)$. This proves that

$$
W \subseteq F(U) \cap Y^{*} .
$$

To prove the reverse inclusion, let $b \in$ $F(U) \cap Y^{*}$. Then $b=f(a)$ for some $a \in X$. We claim that $a \in U$. Suppose that $a \notin U$. Then $U \subseteq$ 
$X-\{a\}$ and hence $F(U) \subseteq F(X-\{a\})$. As $f(a)$ is in $F(U)$ and as $f(a)$ cannot be in $F(X-\{a\})$ we get a contradiction. Thus $a \in U$ and hence $f(a) \in$ $f^{\rightarrow}(U)$. Thus $b \in f^{\rightarrow}(U)$ and hence $b \in W$. This implies that $F(U) \cap Y^{*} \subseteq W$ and hence we get

$$
W=F(U) \cap Y^{*} .
$$

So every member of $\mathcal{T}^{*}$ is of the form $V \cap Y^{*}$ for some $V \in \mathcal{T}^{\prime}$. We shall now prove that every set of the from $V \cap Y^{*}$ for some $V \in \mathcal{T}^{\prime}$ is in $\mathcal{T}^{*}$.

Let $V \in \mathcal{T}^{\prime}$ and let $W=V \cap Y^{*}$. As $F$ is surjective, $V=F(U)$ for some $U \in \mathcal{T}$. We claim that $F^{\rightarrow}(U)=W$. Let $b \in f^{\rightarrow}(U)$. Then $b=f(a)$ for some $a \in U$. Suppose that $f(a) \notin W$. Since $f(a) \in$ $Y^{*}$ we have $f(a) \notin V=F(U)$. But, as $F(X-\{a\})$ is the largest open set not containing $f(a)$, we see that $F(U) \subseteq F(X-\{a\})$ and hence $U \subseteq X-\{a\}$. This implies that $a \notin U$. This is a contradiction to the fact that $a \in U$. So $b=f(a) \in W$ and hence

$$
f^{\rightarrow}(U) \subseteq W .
$$

To prove the reverse inclusion, let $b \in W$. Then $b \in V \cap Y^{*}$. So $b=f(a)$ for some $a \in X$. As $b \in V, f(a) \in V$ and hence $f(a) \in F(U)$. We claim that $a \in U$. Let us assume that $a \notin U$. Then $U \subseteq X-\{a\}$ and hence $F(U) \subseteq F(X-\{a\})$. But as $f(a) \notin F(X-\{a\}), f(a) \notin F(U)$. This is a contradiction to the fact that $f(a) \in F(U)$. This proves that $a \in U$ and hence $b=f(a) \in f^{\rightarrow}(U)$. This shows that

$$
W \subseteq f^{\rightarrow}(U) .
$$

Therefore, $f^{\rightarrow}(U)=W$ which proves that

$$
\mathcal{T}^{*}=\left\{V \cap Y^{*} / V \in \mathcal{T}^{\prime}\right\}
$$

and hence $\left(Y^{*}, \mathcal{T}^{*}\right)$ is a subspace of $\left(Y, \mathcal{T}^{\prime}\right)$.

In the above discussion we have proved that, for all $U \in \mathcal{T}$,

$$
f^{\rightarrow}(U)=F(U) \cap Y^{*} .
$$

Finally we prove that $f$ is a homeomorphism from $(X, \mathcal{T})$ to $\left(Y^{*}, \mathcal{T}^{*}\right)$. As $f$ is a bijection, it remains to prove that $f$ is continuous and it is an open mapping. Let $W \in \mathcal{T}^{*}$. Then $W=V \cap Y^{*}$ for some $V \in \mathcal{T}^{\prime}$. Since $V=F(U)$ for some $U \in \mathcal{T}$, we have $W=F(U) \cap Y^{*}$ for some $U$. So we have $W=$ $f^{\rightarrow}(U)$. This implies that $U=f^{\leftarrow}(W)$ as $f$ is a bijection. This shows that $f$ is continuous. If $U \in \mathcal{T}$, then $f^{\rightarrow}(U)=F(U) \cap Y^{*} \in \mathcal{T}^{*}$. This shows that $f$ is an open mapping. So $f$ is a homeomorphism from $(X, \mathcal{T})$ to $\left(Y^{*}, \mathcal{T}^{*}\right)$.

It is interesting to note that for any $a \in X$, $f(a) \notin F(X-\{a\})$ and $f(a) \in F(X-\{b\})$ for all $b \in X$ other than $a$. The above theorem can be restated as follows:

Theorem 4.4. If two spaces are topomorphic and one point sets in one of the spaces are closed, then the other space contains a subspace homeomorphic to the first space.

Furthermore, if $F:(X, \mathcal{T}) \rightarrow\left(Y, \mathcal{T}^{\prime}\right)$ is a topomorphism and if $(X, \mathcal{T})$ is Hausdorff, then, as one point sets in a Hausdorff space are closed, $Y$ contains a Hausdorff subspace homeomorphic to $(X, \mathcal{T})$. The same is true for all topological properties like regular space and normal space.

As every space is a subspace of itself, the assumption "one point sets are closed" is not a necessary condition in the above theorem. However, the importance of the assumption can be seen from the following example.

Example 4.5. Let $X=Y=\mathbb{R}$. Let

$$
\begin{gathered}
\mathcal{T}_{X}=\{\varnothing,[0,1],[0,2],\{3\},[0,1] \cup\{3\},[0,2] \\
\cup\{3\}, \mathbb{R}\}
\end{gathered}
$$

and

$$
\begin{gathered}
\mathcal{T}_{Y}=\{\varnothing,\{1\},\{1,2\},[3,4],[3,4] \cup\{1\},[3,4] \\
\cup\{1,2\}, \mathbb{R}\} .
\end{gathered}
$$

Then $\left(X, \mathcal{T}_{X}\right)$ and $\left(Y, \mathcal{T}_{Y}\right)$ are topological spaces. They are topomorphic under the mapping which takes the sets of $\mathcal{T}_{X}$ to sets of $\mathcal{T}_{Y}$ in the order we listed them above. But there is neither a subspace of $Y$ homeomorphic to $X$ nor a subspace of $X$ homeomorphic to $Y$.

Let us justify our claim in the example. If possible let $\left(A, \mathcal{T}_{A}\right)$ be a subspace of $Y$ homeomorphic to $\left(X, \mathcal{T}_{X}\right)$. As every member of $\mathcal{T}_{A}$ is of the form $V \cap A$ for some $V \in \mathcal{T}_{Y}$, and as cardinality of $\mathcal{T}_{X}$ and the cardinality of $\mathcal{T}_{A}$ must be equal, $A$ must contain both 1 and 2; for otherwise the cardinality of $\mathcal{T}_{A}$ will be less than that of $\mathcal{T}_{X}$. Let $V=[3,4] \cap A$. Then

$$
\mathcal{T}_{A}=\{\varnothing,\{1\},\{1,2\}, V, V \cup\{1\}, V \cup\{1,2\}, \mathrm{A}\} .
$$

There is no homeomorphism between $\left(A, \mathcal{T}_{A}\right)$ and $\left(X, \mathcal{T}_{X}\right)$ because $\mathcal{T}_{X}$ contains only two finite subsets whereas $\mathcal{T}_{A}$ contains at least three finite subsets.

If possible let $\left(B, \mathcal{T}_{B}\right)$ be a subspace of $X$ homeomorphic to $\left(Y, \mathcal{T}_{Y}\right)$. Then $B$ must contain 3 . Let $C=[0,1] \cap B$ and $D=[0,2] \cap B$. Then

$$
\begin{aligned}
& \mathcal{T}_{B}=\{\varnothing, C, D,\{3\}, C \cup\{3\}, D \cup\{3\}, B\} . \\
& \text { If } f \text { is a homeomorphism from }\left(B, \mathcal{T}_{B}\right) \text { to }
\end{aligned}
$$
$\left(Y, \mathcal{T}_{Y}\right)$, then $f(3)=1$. As $3 \notin C, 3 \notin D$ and $f(3)=1$, either $C \cup\{3\}$ or $D \cup\{3\}$ must be mapped onto $\{1,2\}$. So one of the sets $C$ and $D$ must contain only one point and that point must be 
mapped onto 2 under $f$. Since $C \subseteq D, D$ cannot be an one point set. So $C$ is an one point set and let $C=$ $\{c\}$. So

$$
\mathcal{T}_{B}=\{\varnothing,\{3\},\{c\}, D,\{3, c\}, D \cup\{3\}, B\} .
$$

There is no homeomorphism between $\left(B, \mathcal{T}_{B}\right)$ and $\left(Y, \mathcal{T}_{Y}\right)$ because $\mathcal{T}_{Y}$ contains only three finite subsets whereas $\mathcal{T}_{B}$ contains at least four finite subsets.

Let us consider a converse of Theorem 4.4. If $(X, \mathcal{T})$ and $\left(Y, \mathcal{T}^{\prime}\right)$ are spaces in which one point sets are closed and if each of the spaces has a subspace homeomorphic to the other, can we conclude that there is a topomorphism between the spaces? We cannot conclude so. For example each of the topological spaces $[0,1]$ and $(0,2)$, with usual topology, has uncountably many subspaces homeomorphic to the other; but there is no topomorphism between them by Theorem 3.5 as one is compact and the other is not.

We now prove that a bijection from $X$ to $Y$ which coincides with a topomorphism is necessarily a homeomorphism. So a bijection between two topological spaces preserving arbitrary union and finite intersection of open sets is necessarily a homeomorphism.

Theorem 4.6 Let $F:(X, \mathcal{T}) \rightarrow\left(Y, \mathcal{T}^{\prime}\right)$ be a topomorphism and let $f: X \rightarrow Y$ be a bijection such that

(1) $\quad F(A)=f^{\rightarrow}(A)$ for every $A \in \mathcal{T}$.

Then $f$ is a homeomorphism from $(X, \mathcal{T})$ to $\left(Y, \mathcal{T}^{\prime}\right)$ and the topomorphism induced by $f$ is $F$.

Proof. We first note that (1) is equivalent to

(2) for every $A \in \mathcal{T}$, we have $x \in A$ if and only if $f(x) \in F(A)$

and this equivalent to

(3) for every $B \in \mathcal{T}^{\prime}$, we have $y \in B$ if and only if $f^{-1}(y) \in F^{-1}(B)$.

But by using (3), we see that

(4) $F^{-1}(B)=f^{\leftarrow}(B)$ for every $B \in \mathcal{T}^{\prime}$.

It is easy to see that (1) and (2) prove that $f^{-1}$ and $f$ are continuous as $F(A)$ and $F^{-1}(B)$ are open sets in $\mathcal{T}^{\prime}$ and $\mathcal{T}$. Thus $f$ is a homeomorphism.

\section{Compactness and Connectedness in the Context of Topomorphisms}

Let $(X, \mathcal{T})$ and $\left(Y, \mathcal{T}^{\prime}\right)$ be topological spaces. Let $\mathcal{C}$ and $\mathcal{C}^{\prime}$ denote the collection of all closed sets of $(X, \mathcal{T})$ and $\left(Y, \mathcal{T}^{\prime}\right)$ respectively. With every topomorphism let us associate a function $F_{c}$ from $\mathcal{C}$ to $\mathcal{C}^{\prime}$ and study its properties.
Theorem 5.1. Let $F:(X, \mathcal{T}) \rightarrow\left(Y, \mathcal{T}^{\prime}\right)$ be a topomorphism. Define $F_{C}: \mathcal{C} \rightarrow \mathcal{C}^{\prime}$ as follows:

$$
F_{c}(A)=\left[F\left(A^{c}\right)\right]^{c}
$$

where $A^{c}=X-A$.Then

1. $F_{c}(A \cup B)=F_{c}(A) \cup F_{c}(B)$ for any sets $A$ and $B$ in $\mathcal{C}$.

2. $F_{c}\left(\cap A_{\alpha}\right)=\cap F_{c}\left(A_{\alpha}\right)$ for any collection $\left\{A_{\alpha}\right\}$ of sets in $\mathcal{C}$.

3. $\quad F_{c}$ is a bijection from $\mathcal{C}$ to $\mathcal{C}^{\prime}$ with inverse $F_{c}^{-1}$ defined by

$$
F_{c}^{-1}(B)=\left[F^{-1}\left(B^{c}\right)\right]^{c} .
$$

4. i) $F_{c}(\varnothing)=\emptyset$ and $F_{c}(X)=Y$.

ii) If $A \subseteq B \subseteq X$, then $F_{c}(A) \subseteq F_{c}(B)$; if $A \sqsubseteq$ $B \subseteq X$, then $F_{c}(A) \varsubsetneqq F_{c}(B)$.

iii) If $A \subseteq B \subseteq Y$, then $F_{c}^{-1}(A) \subseteq F_{c}^{-1}(B)$; if $A \varsubsetneqq B \subseteq Y$, then $F_{c}^{-1}(A) \varsubsetneqq F_{c}^{-1}(B)$.

5. i) $F_{c}^{-1}(A \cup B)=F_{c}^{-1}(A) \cup F_{c}^{-1}(B)$ for any sets $A$ and $B$ in $\mathcal{C}^{\prime}$.

ii) $F_{c}^{-1}\left(\cap B_{\alpha}\right)=\cap F_{c}^{-1}\left(B_{\alpha}\right)$ for any collection $\left\{B_{\alpha}\right\}$ of sets in $\mathcal{C}^{\prime}$.

6. i) If $A$ is closed and $B$ is open in $(X, \mathcal{T})$ with $A \subseteq B$, then $F_{c}(A) \subseteq F(B)$.

ii) If $A$ is open and $B$ is closed in $(X, \mathcal{T})$ with $A \subseteq B$, then $F(A) \subseteq F_{c}(B)$.

iii) If $A$ is closed and $B$ is open in $\left(Y, \mathcal{T}^{\prime}\right)$ with $A \subseteq B$, then $F_{c}^{-1}(A) \subseteq F^{-1}(B)$.

iv) If $A$ is open and $B$ is closed in $\left(Y, \mathcal{T}^{\prime}\right)$ with $A \subseteq B$, then $F^{-1}(A) \subseteq F_{c}^{-1}(B)$.

Proof.

$$
\begin{gathered}
F_{c}(A \cup B)=\left[F\left((A \cup B)^{c}\right)\right]^{c}=\left[F\left(A^{c} \cap B^{c}\right)\right]^{c} \\
=\left[F\left(A^{c}\right) \cap F\left(B^{c}\right)\right]^{c} \\
=\left[F\left(A^{c}\right)\right]^{c} \cup\left[F\left(B^{c}\right)\right]^{c}=F_{c}(A) \cup F_{c}(B) .
\end{gathered}
$$

This proves (1); (2) can be proved similarly.

Let $A \in \mathcal{C}$.

$$
\begin{gathered}
F_{c}^{-1}\left(F_{c}(A)\right)=\left(F^{-1}\left[\left(F_{c}(A)\right)^{c}\right]\right)^{c} \\
=\left(F^{-1}\left[\left(\left[F\left(A^{c}\right)\right]^{c}\right)^{c}\right]\right)^{c} \\
=\left(F^{-1}\left[F\left(A^{c}\right)\right]\right)^{c}=\left(A^{c}\right)^{c} \\
=A .
\end{gathered}
$$

Similarly for $B \in \mathcal{C}^{\prime}, F_{c}\left(F_{c}^{-1}(B)\right)=B$ and hence $F_{C}$ is a bijection whose inverse is given by $F_{c}^{-1}(B)=\left[F^{-1}\left(B^{c}\right)\right]^{c}$. Thus (3) follows.

The identities $F_{c}(\varnothing)=\varnothing$ and $F_{c}(X)=Y$ follow from the definition.

If $A \subseteq B \subseteq X$, then $B^{c} \subseteq A^{c}$ and hence $F\left(B^{c}\right) \subseteq F\left(A^{c}\right)$. This implies that $\left(F\left(A^{c}\right)\right)^{c} \subseteq$ 
$\left(F\left(B^{c}\right)\right)^{c}$ and hence $F_{c}(A) \subseteq F_{c}(B)$. The other results in (4) follow similarly.

All results in (5) follow from the definition.

Let $A$ be open and $B$ be closed in $(X, \mathcal{T})$ and let $A \subseteq B$. Since $A \subseteq B, A \cap B^{c}=\emptyset$; thus $F(A \cap$ $\left.B^{c}\right)=\varnothing$ and hence $F(A) \cap F\left(B^{c}\right)=\emptyset$; thus $F(A) \subseteq\left(F\left(B^{c}\right)\right)^{c}$ and hence $F(A) \subseteq F_{c}(B)$. Other results in (6) follow similarly.

Theorem 5.2. Let $F:(X, \mathcal{T}) \rightarrow\left(Y, \mathcal{T}^{\prime}\right)$ be a topomorphism. If $A$ is open and closed, then

$$
F(A)=F_{c}(A)=\left(F\left(A^{c}\right)\right)^{c}=\left(F_{c}\left(A^{c}\right)\right)^{c} .
$$

Proof. Let $B=A^{C}$. Since $A$ and $B$ are complement to each other, $F(A)$ and $F(B)$ are complement to each other. Thus

$$
F(A)=(F(B))^{c}=\left(F\left(A^{c}\right)\right)^{c} .
$$

Other results follow similarly.

Theorem 5.3. Let $F:(X, \mathcal{T}) \rightarrow\left(Y, \mathcal{T}^{\prime}\right)$ be a topomorphism. If $A$ is a closed compact set in $X$, then $F_{c}(A)$ is compact in $Y$.

Proof. Since $A$ is closed, $F_{c}(A)$ is meaningful and is closed in $\left(Y, \mathcal{T}^{\prime}\right)$. Let $\left\{V_{\alpha}\right\}$ be an open cover for $F_{c}(A)$. For all $\alpha$ let $U_{\alpha}=F^{-1}\left(V_{\alpha}\right)$. We claim that $\left\{U_{\alpha}\right\}$ is a cover for $A$. Now

$$
F_{c}(A) \subseteq \cup V_{\alpha}=\cup F\left(U_{\alpha}\right)=F\left(\cup U_{\alpha}\right) \text {. }
$$

Thus by (6) of Theorem 5.1,

$$
F_{c}^{-1}\left(F_{c}(A)\right) \subseteq F^{-1}\left(F\left(\cup U_{\alpha}\right)\right)
$$

and hence

$$
A \subseteq \cup U_{\alpha} .
$$

Thus $\left\{U_{\alpha}\right\}$ is an open cover for $A$ and hence has a finite subcover, say $\left\{U_{1}, U_{2}, \ldots, U_{n}\right\}$. That is

$$
A \subseteq U_{1} \cup U_{2} \cup \ldots \cup U_{n} .
$$

Therefore

$$
\begin{aligned}
F_{c}(A) & \subseteq F\left(U_{1} \cup U_{2} \cup \ldots \cup U_{n}\right) \\
& \subseteq F\left(U_{1}\right) \cup F\left(U_{2}\right) \cup \ldots \cup F\left(U_{n}\right) \\
& =V_{1} \cup V_{2} \cup \ldots \cup V_{n} .
\end{aligned}
$$

Thus $\left\{V_{1}, V_{2}, \ldots, V_{n}\right\}$ is a finite subcover for $F_{c}(A)$ and hence $F_{c}(A)$ is compact.

Corollary 5.4. Let $(X, \mathcal{T})$ be a Hausdorff space and $F:(X, \mathcal{T}) \rightarrow\left(Y, \mathcal{T}^{\prime}\right)$ be a topomorphism. If $A$ is a compact subset of $X$, then $F_{C}(A)$ is compact in $Y$.

Theorem 5.5. Let $F:(X, \mathcal{T}) \rightarrow\left(Y, \mathcal{T}^{\prime}\right)$ be a topomorphism. i. If $A$ is an open connected subset of $X$, then $F(A)$ is a connected subset of $Y$.

ii. If $A$ is a closed connected subset of $X$, then $F_{c}(A)$ is a connected subset of $Y$.

iii. If $A$ is a connected subset of $X$, then $F_{c}(\bar{A})$ is a connected subset of $Y$ where $\bar{A}$ denotes the closure of $A$ in $X$.

Proof. We prove the third one, as the other results follow similarly. Since $\bar{A}$ is closed, $F_{c}(\bar{A})$ is meaningful and is closed in $\left(Y, \mathcal{T}^{\prime}\right)$. As $A$ is connected, $\bar{A}$ is also connected. If $F_{c}(\bar{A})$ is not connected, then there exist nonempty disjoint sets $C$ and $D$, closed in $F_{c}(\bar{A})$ such that $F_{c}(\bar{A})=C \cup D$. As $F_{C}(\bar{A})$ is closed in $Y, C$ and $D$ are closed in $Y$ also. So we get

$$
\bar{A}=F_{c}^{-1}\left(F_{c}(\bar{A})\right)=F_{c}^{-1}(C) \cup F_{c}^{-1}(D)
$$

which implies that $\bar{A}$ is not connected. This is a contradiction to the fact that $\bar{A}$ is connected. This completes the proof.

\section{TOPOMORPHISMS AND BASES FOR TOPOLOGIES}

In this section we discuss topomorphism in the context of basis for a topology.

Theorem 6.1. Let $F:(X, \mathcal{T}) \rightarrow\left(Y, \mathcal{T}^{\prime}\right)$ be a topomorphism and $\mathfrak{B}$ be a basis for $\mathcal{T}$. Then $\mathfrak{B}^{\prime}=$ $F(\mathfrak{B})$ is a basis for $\mathcal{T}^{\prime}$ where $F(\mathfrak{B})=\{F(B) /$ $B \in \mathfrak{B}\}$.

Proof. Since $\cup_{B \in \mathfrak{B}} B=X$, we have

$$
F\left(\cup_{B \in \mathfrak{B}} B\right)=F(X)
$$

and hence

$$
\cup_{B \in \mathfrak{B}} F(B)=\cup_{B^{\prime} \in \mathfrak{B}^{\prime}} B^{\prime}=Y .
$$

Suppose that $y \in F\left(B_{1}\right) \cap F\left(B_{2}\right)$. Then $C=$ $F\left(B_{1}\right) \cap F\left(B_{2}\right)$ is an open set in $\left(Y, \mathcal{T}^{\prime}\right)$ and hence $F^{-1}(C)$ is an open set in $(X, \mathcal{T})$. Thus

$$
F^{-1}(C)=\cup_{\alpha \in \Lambda} B_{\alpha}
$$

for some indexing set $\Lambda$. This implies that

$$
C=F\left(\cup_{\alpha \in \Lambda} B_{\alpha}\right)=\cup_{\alpha \in \Lambda} F\left(B_{\alpha}\right) .
$$

Therefore $y \in F\left(B_{\alpha_{0}}\right)$ for some $\alpha_{0}$. Hence

$$
y \in F\left(B_{\alpha_{0}}\right) \subseteq C=F\left(B_{1}\right) \cap F\left(B_{2}\right) .
$$

If $V$ is an open set in $\left(Y, \mathcal{T}^{\prime}\right)$, then $F^{-1}(V)$ is open in $(X, \mathcal{T})$ and hence $F^{-1}(V)=\cup B_{\alpha}$. This implies that $V=F\left(\cup B_{\alpha}\right)=\cup F\left(B_{\alpha}\right)$. Thus $F(B)$ is a basis for $\mathcal{T}^{\prime}$. 
Theorem 6.2. Let $X$ and $Y$ be nonempty sets, and let $\mathfrak{B}$ and $\mathfrak{B}^{\prime}$ be bases for topologies $\mathcal{T}$ and $\mathcal{T}^{\prime}$ on $X$ and $Y$ respectively. Let $F$ be a function from $\mathfrak{B}$ onto $\mathfrak{B}^{\prime}$ having the following properties.

(1) If $B_{1} \cap B_{2} \subseteq \cup_{\alpha \in \Lambda} B_{\alpha}$, then $F\left(B_{1}\right) \cap$ $F\left(B_{2}\right) \subseteq \cup_{\alpha \in \Lambda} F\left(B_{\alpha}\right)$.

(2) If $F(B) \subseteq \cup_{\alpha \in \Lambda} F\left(B_{\alpha}\right)$, then $B \subseteq \cup_{\alpha \in \Lambda} B_{\alpha}$.

Then $F$ can be extended to a topomorphism from $\mathcal{T}$ to $\mathcal{T}^{\prime}$ uniquely.

Proof. We first note that (1) implies that

$$
\begin{aligned}
& \text { If } B \subseteq \cup_{\alpha \in \Lambda} B_{\alpha}, \text { then } F(B) \subseteq \\
& \cup_{\alpha \in \Lambda} F\left(B_{\alpha}\right) \text {. } \\
& \text { If } B_{1} \subseteq B_{2} \text {, then } F\left(B_{1}\right) \subseteq F\left(B_{2}\right) .
\end{aligned}
$$

Define $G: \mathcal{T} \rightarrow \mathcal{T}^{\prime}$ as follows:

$$
G(V)=\cup F\left(B_{\alpha}\right)
$$

where $V=\cup B_{\alpha}$.

We first claim that $G$ is well defined. Suppose $\mathrm{U}_{\alpha \in \Lambda} B_{\alpha}=\mathrm{U}_{\beta \in \Lambda^{\prime}} B_{\beta}$. Since $B_{\alpha} \subseteq \mathrm{U}_{\beta \in \Lambda^{\prime}} B_{\beta}$, by using (3) we have

$$
F\left(B_{\alpha}\right) \subseteq \cup_{\beta \in \Lambda^{\prime}} F\left(B_{\beta}\right)
$$

and hence

$$
\mathrm{U}_{\alpha \in \Lambda} F\left(B_{\alpha}\right) \subseteq \mathrm{U}_{\beta \in \Lambda^{\prime}} F\left(B_{\beta}\right)
$$

Similarly we get

and hence

$$
\mathrm{U}_{\beta \in \Lambda^{\prime}} F\left(B_{\beta}\right) \subseteq \mathrm{U}_{\alpha \in \Lambda} F\left(B_{\alpha}\right)
$$

$$
\cup_{\alpha \in \Lambda} F\left(B_{\alpha}\right)=\cup_{\beta \in \Lambda^{\prime}} F\left(B_{\beta}\right)
$$

This shows that $G$ is well defined.

Now we claim that $G$ is a topomorphism. Let $\left\{V_{\alpha}\right\}_{\alpha \in \Lambda}$ be a collection of open sets in $(X, \mathcal{T})$. Let

$$
V_{\alpha}=\cup_{\lambda \in \Lambda_{\alpha}} B_{\alpha, \lambda} .
$$

Then

$$
\begin{aligned}
G\left(\cup_{\alpha \in \Lambda} V_{\alpha}\right) & =G\left(\cup_{\alpha \in \Lambda} \cup_{\lambda \in \Lambda_{\alpha}} B_{\alpha, \lambda}\right) \\
& -\cup_{\alpha \in \Lambda} \cup_{\lambda \in \Lambda_{\alpha}} F\left(B_{\alpha, \lambda}\right) \\
& =\cup_{\alpha \in \Lambda} G\left(V_{\alpha}\right) .
\end{aligned}
$$

Now let $A=\cup_{\alpha \in \Lambda} B_{\alpha}$ and $B=\cup_{\beta \in \Lambda^{\prime}} B_{\beta}$. Then

$$
\begin{aligned}
G(A) \cap G(B) & =\left(\cup_{\alpha \in \Lambda} F\left(B_{\alpha}\right)\right) \cap\left(\cup_{\beta \in \Lambda^{\prime}} F\left(B_{\beta}\right)\right) \\
& =\cup_{\alpha \in \Lambda} \cup_{\beta \in \Lambda^{\prime}}\left(F\left(B_{\alpha}\right) \cap F\left(B_{\beta}\right)\right) \\
& \subseteq \cup_{\alpha \in \Lambda} \cup_{\beta \in \Lambda^{\prime}}\left(\cup_{\gamma \in \Gamma_{\alpha, \beta}} F\left(B_{\alpha, \beta, \gamma}\right)\right)
\end{aligned}
$$

where

$$
\begin{aligned}
& B_{\alpha} \cap B_{\beta}=\cup_{\gamma \in \Gamma_{\alpha, \beta}} B_{\alpha, \beta}, \gamma \\
& \quad=G(A \cap B)
\end{aligned}
$$

since $A \cap B=\cup_{\alpha \in \Lambda} \cup_{\beta \in \Lambda^{\prime}} \cup_{\gamma \in \Gamma_{\alpha, \beta}} B_{\alpha, \beta}, \gamma$.

By using (4), we see that for $A, B \in \mathcal{T}$, if $A \subseteq$ $B$, then $G(A) \subseteq G(B)$. Since $A \cap B \subseteq A$, we have $G(A \cap B) \subseteq G(A)$; similarly we have $G(A \cap B) \subseteq$ $G(B)$. Thus $G(A \cap B) \subseteq G(A) \cap G(B)$ and hence we have $G(A \cap B)=G(A) \cap G(B)$.

Let $W \in \mathcal{T}^{\prime}$. Since $\mathfrak{B}^{\prime}$ is a basis for $\mathcal{T}^{\prime}$ we have $W=\cup_{\alpha \in \Lambda} B_{\alpha}^{\prime}$ for some subcollection $\left\{B_{\alpha}^{\prime}\right\}_{\alpha \in \Lambda}$ of $\mathcal{T}^{\prime}$. Since $F$ is onto, for all $\alpha \in \Lambda$, there exists, $B_{\alpha}$ such that $B_{\alpha}^{\prime}=F\left(B_{\alpha}\right)$. If $V=\cup_{\alpha \in \Lambda} B_{\alpha}$, then $G(V)=W$. Thus $G$ is onto.

To prove $G$ is one-to-one, let $V_{1}, V_{2} \in \mathcal{T}$ and $G\left(V_{1}\right)=G\left(V_{2}\right)$. Let

$$
V_{1}=\cup_{\alpha \in \Lambda} B_{\alpha} \text { and } V_{2}=\cup_{\beta \in \Lambda^{\prime}} B_{\beta} .
$$

For $\alpha \in \Lambda$,

$$
\begin{gathered}
F\left(B_{\alpha}\right) \subseteq \cup_{\alpha \in \Lambda} F\left(B_{\alpha}\right)=G\left(V_{1}\right)=G\left(V_{2}\right) \\
=\cup_{\beta \in \Lambda^{\prime}} F\left(B_{\beta}\right) .
\end{gathered}
$$

Thus by (2), $B_{\alpha} \subseteq \cup_{\beta \in \Lambda^{\prime}} B_{\beta}$ and hence $V_{1} \subseteq$ $V_{2}$. Similarly $V_{2} \subseteq V_{1}$ and hence $V_{1}=V_{2}$ which implies that $G$ is one-to-one. Thus $G$ is a topomorphism.

To prove the uniqueness let $G^{\prime}$ be a topomorphism and $V=\cup B_{\alpha} \in \mathcal{T}$, then

$$
\begin{gathered}
G^{\prime}(V)=G^{\prime}\left(\cup B_{\alpha}\right)=\cup G^{\prime}\left(B_{\alpha}\right)=\cup F\left(B_{\alpha}\right) \\
=G(V) .
\end{gathered}
$$

This implies that the extension is unique.

It is easy to see that if the condition "onto" on $F$ is removed from the statement, then $(X, \mathcal{T})$ will be topomorphic to a subspace of $\left(Y, \mathcal{T}^{\prime}\right)$ which is formed by taking the union $\cup F(B)$ of all sets $B \in$ $\mathfrak{B}$.

\section{CONCLUSION}

We have defined and discussed a new concept called topomorphism, as a bijection between topologies which preserve finite intersection and arbitrary union. Topomorphisms in the context of connectedness, compactness, separation axioms and the role of basis in topomorphism were studied deeply. A good theory may be developed by relaxing the condition "bijection" in the definition of topomorphism. Further, a similar theory can be developed in the fuzzy topology theory. Topomorphism do not identify discrete topological spaces on finite sets. A parallel theory may be developed in this context.

\section{REFERENCES}

[1] J. R. Munkres, Topology, Prentice-Hall of India, Second Edition, 2006. 
ТОПОМОРФИЗМИ - НОВ ПРИСТАП КОН ИДЕНТИФИКУВАЊЕ НА ТОПОЛОГИИ

\section{Ramachandran Vembu}

Department of Mathematics, SBK College, Aruppukottai - 626 101, India

Во овој труд е воведен и дискутиран нов концепт наречен топоморфизам. Топоморфизам се дефинира како биекција меѓу топологии која запазува конечни пресеци и произволни унии. Докажано е дека топоморфизмите запазуваат сврзаност и компактност. Истражувани се топоморфизмите во контекст на аксиомите за одделување, како и улогата на бази за топоморфизми.

Клучни зборови: Топологија; хомеоморфизам; топоморфизам; идентификација на топологии; сврзаност; компактност; аксиоми за одделување 